\title{
¿QUÉ HACEN LOS DOCENTES EN SUS AULAS? EL CASO DE UN CENTRO DE EDUCACIÓN PRIMARIA
}

\author{
$M^{a}$ Isabel Robles Gómez \\ $M^{a}$ Begoña Alfageme González \\ Mónica Vallejo Ruiz \\ Universidad de Murcia
}

RESUMEN: El presente artículo describe la realidad de siete docentes de Educación Primaria en relación al uso de metodologías didácticas en sus aulas. Este estudio se centra en analizar las metodologías Ilevadas a cabo por docentes de Primaria para conocer el grado de implantación del modelo basado en competencias en este nivel educativo. La actualidad del tema y la falta de investigaciones en este nivel educativo motivan la realización de tal estudio. Concretamente, se trata de un estudio de caso de carácter descriptivo realizado en un centro de Educación Primaria en la Región de Murcia. Así, a través de entrevistas semiestructuradas, analizamos la práctica real de aula que emplean siete docentes -cinco mujeres y dos hombres de distintos niveles y especialidades-, para conocer cómo imparten sus clases.

Las conclusiones apuntan a que los entrevistados aún no tienen asumido un modelo educativo basado en competencias, aunque se vislumbran indicios de una nueva reorientación de las metodologías empleadas y actividades desarrolladas con el objeto de la consecución de las ocho competencias básicas. Así, parece que los docentes se encuentran en un primer estadio de adaptación a la nueva concepción de los enfoques de enseñanza y aprendizaje.

ABSTRACT: This paper presents an investigation focusing on the need to analyze the methodologies undertaken by primary school teachers to see the implementation of competency-based model at this level. The lack of research in this respect the basic levels of education in our country makes this study relevant.

Specifically it is a descriptive case study conducted in a Primary School. Thus, through semi-structured interviews, we discuss the real classroom practice employing seven teachers to see how their teaching, five women and two men from different levels and specialties. 
The findings suggest that the teachers interviewed have not yet taken a competency-based educational model, but there are emerging signs of a new shift in media and methodologies used. This indicates that teachers are trying to adapt to new demands of continual change.

PALABRAS CLAVE: Educación Primaria, metodologías, competencias básicas, estudio descriptivo, análisis de aula.

KEYWORDS: Primary School, teachers, methodologies, key competences, descriptive study, analysing classroom.

Recibido: $31 / 03 / 2010$

Aceptado: 02/12/2010

\section{INTRODUCCIÓN}

Las innovaciones se suceden en educación cada vez a un mayor ritmo. En este sentido, las reformas y los cambios se han ido convirtiendo en "amigos inseparables" de los sistemas escolares (Escudero, 1990).

Los cambios sociales, cada vez más profundos y extensos, siguen poniendo en cuestión el modelo clásico de institución escolar; siendo ésta sometida a un proceso complejo de reorientación de sus fines y medios para intentar adaptarse a las demandas y desafíos de la sociedad actual.

Particularmente, se están produciendo importantes cambios en los currículos de todos los niveles de enseñanza. La Unión Europea ha establecido, por vez primera, que el currículo incluya unas competencias básicas para facilitar el aprendizaje permanente. El Real Decreto 1513/2006 de Enseñanzas Mínimas de Educación Primaria (BOE, 2006) establece que desde las distintas áreas se debe contribuir a la adquisición y desarrollo de ocho competencias básicas. Se concibe desde un enfoque integrador e interdisciplinar, que contribuye a transformar el concepto tradicional de enseñanza basado en la adquisición de conocimientos, en un concepto de aprendizaje cimentado en la capacidad del propio alumno para resolver situaciones a lo largo de la vida. Además, en la Región de Murcia, estas competencias han pasado a ser elementos integrantes de los nuevos currículos y afectarán a los distintos elementos que conforman el proceso de enseñanza-aprendizaje, tanto en la planificación como en el desarrollo de la práctica docente de cada una de las materias, ya que es en cada una de ellas donde el alumno tiene que haber adquirido el desarrollo de dichas competencias antes de finalizar la enseñanza obligatoria. Por ello, se ha introducido en el sistema escolar un planteamiento de aprendizaje basado en competencias que imprime un nuevo rumbo en la práctica docente. La Educación ya no se restringe a las aulas, sino que se enmarca en un contexto más amplio donde el sujeto debe ser capaz de "manipular" el conocimiento, ponerlo al día, seleccionarlo, aprender de forma permanente y entenderlo; y todo ello de forma que pueda adaptarlo a nuevas situaciones que tan rápido se transforman. Por tanto, la introducción de un aprendizaje basado en competencias ha "complicado" las funciones más tradicionales de los docentes, que deben considerar la necesidad de ir pasando de una enseñanza centrada, de forma exclusiva, en la transmisión de conocimientos, a otra que se centre en el aprendizaje del alumno favoreciendo sus capacidades y potencialidades, de manera que se contribuya a lograr su desarrollo integral (Murillo, 2007). 
Este nuevo papel del docente implica la reformulación y el replanteamiento de la metodología y de la organización de la docencia, entre otros aspectos. Pero, a su vez, la consolidación de estos cambios requiere de un proceso complejo de asunción de nuevas concepciones y enfoques; y, sobre todo, de una nueva práctica pedagógica. Éste es el motivo central por el cual consideramos importante conocer cuál es la realidad de nuestras aulas y tomar conciencia de lo que se está haciendo, cómo se está haciendo y porqué.

No olvidemos que como todo cambio, la introducción del modelo de competencias en la enseñanza corre el riesgo de quedarse exclusivamente en una reformulación, adecuando el lenguaje, pero manteniendo las prácticas; es decir, transformándose en un mero ejercicio de ajuste normativo-burocrático que no tenga trascendencia alguna en la práctica educativa. Sobre todo, teniendo en cuenta que las innovaciones en nuestro sistema educativo se han impuesto, olvidándose de que: (...) los cambios impuestos tienen poca, por no decir ninguna, incidencia en las organizaciones educativas (Murillo, 2007: 5).

Para que los cambios tengan una incidencia real en la vida de los centros han de generarse desde su realidad, y han de capacitar para el desarrollo de una cultura innovadora propia que incida en su organización y profesionalización. Sólo así lograremos la implicación del profesorado en un análisis reflexivo de lo que hace.

A este respecto, planteamos la necesidad de analizar las metodologías llevadas a cabo por los docentes para conocer dicha implantación. Así, en este estudio hemos indagado sobre el trabajo realizado en las aulas de siete docentes de Educación Primaria que trabajan en un centro de la Región de Murcia.

\section{Delimitación conceptual}

Como sabemos, el modelo de enseñanza por competencias surge con el objetivo de lograr una mayor cohesión y unidad entre los países que forman la Unión Europea. Dicho modelo supone un giro radical en la concepción del proceso de enseñanza y aprendizaje; entendiéndose las competencias como un conjunto de destrezas, conocimientos, habilidades, actitudes, valores éticos y emociones adecuados al contexto que el alumnado debe alcanzar para su realización, desarrollo personal e integración social.

Tal y como aparece en el R.D. 1513/2006, de 7 de diciembre, por el que se establecen las enseñanzas mínimas de la Educación Primaria, la incorporación de las competencias básicas al currículo permite poner el acento en los aprendizajes que se consideran imprescindibles, desde un planteamiento integrador y orientado a la aplicación de los saberes adquiridos. La inclusión de las competencias básicas en el currículo tiene varias finalidades y permitirá al alumno: lograr una realización personal, ejercer una ciudadanía activa e incorporarse a la vida adulta de manera satisfactoria, siendo capaz de desarrollar un aprendizaje permanente.

De modo sintético, algunos de los rasgos principales de este modelo educativo serían: enfoca el proceso de aprendizaje-enseñanza como trabajo cooperativo entre docentes y alumnos, centrado en el aprendizaje autónomo del estudiante; exige una nueva definición de las actividades de aprendizaje-enseñanza; propone una nueva organización del aprendizaje y plantea la necesidad de una evaluación auténtica. 
Dichos rasgos exigen el desarrollo de un perfil profesional, de unos roles y unas actividades diferentes a las tradicionales en estudiantes y profesores (Fernández March, 2006). Este nuevo papel implica la reformulación de objetivos y de contenidos de aprendizaje, para definir habilidades específicas y transversales, el replanteamiento de la metodología y de la organización de la docencia, pero también el diseño y la concreción de tareas como núcleo del proceso.

El cambio de modelo educativo hacia el que nos dirigimos determina, de algún modo, el tipo de metodologías o estrategias de aprendizaje-enseñanza más pertinentes para alcanzar los rasgos anteriormente descritos.

En síntesis, el reto está en el diseño de metodologías activas que favorezcan la formación en competencias, que sitúen al alumno en una posición diferente a la habitual, pues él mismo ha de ser responsable de su propio aprendizaje: buscando, seleccionando, analizando y evaluando la información; en definitiva, asumiendo un papel más activo.

Así, por tanto, la metodología ha pasado a ser uno de los focos de atención más indagados en estos últimos años. Un ejemplo de ello es la ingente producción que ha surgido en torno a la Educación Superior (véase De Miguel, 2005, 2006; Rodríguez Izquierdo, 2008; Garrote, 2008; Vallejo y Alfageme, 2009). Sin embargo, aunque el tema es relevante y actual en el ámbito de la Educación Superior, no lo es tanto en la etapa de Primaria. Concretamente en esta etapa, los estudios se han centrado en "innovaciones metodológicas" -expresión utilizada en algunos de los estudios analizados- concretas y circunscritas a una materia. Éste es el caso del estudio de Bermejo (2006) en el que se reflexiona acerca del uso de metodologías de enseñanza-aprendizaje erróneas que suelen utilizar algunos profesores, a la vez que hace una llamada a un cambio metodológico que debe venir apoyado por una constante formación del profesorado. Paredes Labra (1999) explora las posibilidades y usos de los recursos y materiales didácticos en Educación Primaria en el marco de un estudio sobre el pensamiento del profesor. Woolf y Lohman (2001) realizan un estudio sobre las actividades docentes en colegios públicos de Estados Unidos; concretamente, son examinadas las actividades de auto-iniciación al aprendizaje de profesores veteranos de escuelas públicas. Estos autores concluyen que la utilización de determinadas metodologías está influenciada por la disponibilidad de recursos, así como por el entorno físico y social. Por otro lado, Murillo (2007) realiza una investigación centrada en la importancia de optar por metodologías activas que fomenten la colaboración entre los alumnos.

\section{Estudio EMPÍRICO}

Como ya hemos comentado, los hechos que justifican esta investigación tienen su base en los cambios que se están dando en todos los niveles de enseñanza mediante la introducción de competencias básicas en el currículo y, más concretamente, en Educación Primaria. La inclusión de las competencias básicas dentro del currículo supone una serie de cambios en los distintos elementos que lo componen, siendo la metodología uno de los componentes clave e imprescindible a adaptar a la nueva realidad educativa. 
Somos conscientes de la dificultad que genera esta compleja tarea, del considerable esfuerzo que realizan los docentes por adaptarse a la nueva realidad, además del riesgo que supone reformular planes adecuando el lenguaje e introduciendo nuevas orientaciones técnicas generadas por el modelo de enseñanza basado en competencias, manteniendo la realidad de nuestras aulas vinculada a los planteamientos metodológicos clásicos, con un enfoque centrado en el docente.

Para evitar que suceda esto, consideramos necesario realizar estudios orientados a generar y facilitar un cambio de modelo en los procesos de enseñanza. La investigación que se describe tiene su justificación dentro de esta línea de trabajo, planteando como objetivo central el analizar y conocer las metodologías que se están utilizando en la actualidad en los centros educativos para que, partiendo de la realidad docente, se introduzcan los cambios necesarios de forma contextualizada. Específicamente se analizan las diferentes metodologías desarrolladas por los docentes de un centro católico concertado de Educación Primaria en la Región de Murcia, entendiendo que la metodología es un buen indicador para definir una determinada concepción de la enseñanza.

Así, el análisis de la misma nos permitirá determinar si se está desarrollando un enfoque de enseñanza con un carácter más tradicional (centrado en el docente) o un enfoque que otorgue un papel más activo al alumno. Igualmente, permitirá conocer la realidad de la práctica docente en el día a día de nuestros centros educativos.

La muestra está compuesta por 7 docentes de Educación Primaria, que constituyen el 30\% de los docentes del centro. Dicha muestra acoge docentes de los tres ciclos de Primaria, cinco de los cuáles son mujeres y dos hombres, con edades comprendidas entre los 24 y 55 años. Los docentes han sido seleccionados mediante un muestreo intencional, quedando establecido como criterio, que cada uno de ellos fuese de un nivel educativo y de especialidad diferente.

Nuestro diseño metodológico trata de combinar diferentes técnicas y estrategias, situadas dentro de los denominados métodos descriptivos. Más concretamente, se trata de un estudio de casos, de carácter eminentemente descriptivo, en el que se persigue describir de forma sistemática la realidad educativa de unos docentes, analizando las metodologías didácticas empleadas en sus aulas y las implicaciones subyacentes a ellas.

Se ha utilizado como instrumento, para la recogida de la información, una entrevista semiestructurada. En ella se ha desarrollado un listado de dimensiones generales que debían cubrirse con el fin de asegurar que los temas clave de este estudio fuesen explorados con cada informante (siguiendo a Blasco y Otero, 2008). Así pues, no se establecía un orden prefijado de preguntas sino que la finalidad de dicho guión era asegurarnos que cada uno de los docentes entrevistados nos facilitase información sobre todas las dimensiones planteadas.

Con relación al tratamiento y el análisis de los datos se ha realizado análisis de tipo cualitativo y cuantitativo. El análisis cualitativo, siguiendo el enfoque ya clásico propuesto por Miles y Huberman (1984), consistió en dar sentido a la información textual recogida a través de las entrevistas. En este sentido, no partimos de una definición previa de categorías, sino que se elaboraron a partir del significado de las anotaciones tomadas por las investigadoras, estableciendo categorías preliminares. 
A excepción de la información referida a metodologías y a actividades, que se basaron en clasificaciones ya realizadas por otros autores (Fernández March, 2006; De Miguel, 2005, 2006; Murrillo, 2007). Posteriormente, se realizó un proceso de reducción de datos quedando como resultante un mapa de significados. Finalmente, pasamos a diseñar un cuadro que contenía todas las categorías y subcategorías establecidas para cada pregunta y docente.

El análisis cuantitativo se centró, por un lado, en el tratamiento de los datos identificativos y, por otro, en el recuento de las categorías elaboradas para poder interpretar los resultados con la finalidad de desvelar la estructura subyacente a las respuestas de los docentes, utilizando para ello el paquete estadístico SPSS-15.

\section{ANÁLISIS DE RESULTADOS}

\section{Datos de identificación}

Los docentes que han participado en este estudio han sido 5 mujeres y 2 hombres, con edades comprendidas entre los 24 a 55 años, si bien el mayor número de sujetos entrevistados se sitúa en el intervalo de 41-50 años.

En cuanto a la experiencia docente, los datos revelan que tres docentes tienen entre 1 y 11 años de experiencia, dos de 21 a 30 y otros dos de 31 a 40 años. En general, los docentes de la muestra no han permanecido mucho tiempo en el centro educativo (cuatro docentes llevan entre 0 a 3 años, dos entre 7 y 9 años y uno más de 12 años); existiendo una amplia movilidad docente.

En relación a las materias que imparten, señalar que éstas son muy diversas; siendo cuatro los maestros generalistas de Educación Primaria que imparten las materias propias de Primaria: Conocimiento del medio, Lengua castellana y Literatura, Matemáticas y Educación artística (plástica), a las que se suma Religión Católica. Por otro lado, los docentes especialistas serían los que imparten las materias de sus especialidades: Lengua extranjera (Inglés) y Educación artística (Música); y, por último, la especialista en Educación Especial que imparte clases de Matemáticas, Lengua Castellana y Literatura y Conocimiento del Medio.

En este sentido, y teniendo en cuenta esta información, creemos que a pesar de que la muestra es escasa, sí que refleja la heterogeneidad de niveles y especialidades dentro de la Educación Primaria. Todos estos datos aportarán el escenario sobre el que contextualizar el resto de la información recogida que se presenta en torno a cuatro grandes bloques.

\section{a) Primer bloque: Objetivos}

Para conocer los objetivos que planteaban los docentes respecto a su práctica profesional se realizaron, en el primer apartado de la entrevista, dos preguntas, una referente al objetivo final de su práctica docente y otra a la programación docente.

Con relación a los objetivos, los datos recogidos apuntan a que 6 de los docentes entrevistados centran su enseñanza en los objetivos curriculares establecidos para esta etapa. Por otro lado, todos los docentes han señalado objetivos de tipo personal (Ej. Lograr que los alumnos sean personas de provecho. Docente 3); y, sola- 
mente uno de ellos, señala objetivos de tipo profesional. En el primer caso, objetivos curriculares, los datos nos indican que 4 docentes señalan como prioridad "superar y conseguir los objetivos y contenidos curriculares", mientras que los otros tres indican enfocar su enseñanza a "aprendizajes funcionales e instrumentales". Sólo dos docentes dan sendas respuestas en su intervención. En relación a los objetivos personales, hemos recogido un total de 7 categorías tras analizar los datos (ver tabla 1). Entre dichos datos destaca el hecho de que la categoría con más aportaciones ha sido la referente a "educar a los alumnos en valores y actitudes", seguida muy de cerca por otras como "que el niño esté a gusto en clase" o "que se forme como persona y se prepare para desenvolverse autónomamente en la vida".

Tabla 1. Categorías referentes a los objetivos personales que señalan los docentes

\begin{tabular}{|l|c|}
\hline & Total \\
\hline Darle una educación en valores y actitudes & 5 \\
\hline Que el niño esté a gusto en clase y en el centro. Motivado & 4 \\
\hline Que se forme como persona & 4 \\
\hline Que se les prepare para desenvolverse autónoma y adecuadamente en la vida & 4 \\
\hline Conseguir una educación integral del alumno & 3 \\
\hline Adquisición de hábitos de trabajo y de estudio, responsabilidades & 3 \\
\hline $\begin{array}{l}\text { Que se acepten a sí mismos tal y como son, sean felices y tengan una } \\
\text { autoestima alta }\end{array}$ & 3 \\
\hline
\end{tabular}

Con la segunda cuestión, dentro del bloque de objetivos, respecto a la elaboración de la programación, perseguíamos determinar cómo trabajan y, por lo tanto, si dicho trabajo lo realizan poniendo en práctica métodos más colaborativos y participativos que emergen frente a los más tradicionales. Como podemos observar en la tabla 2, los resultados nos indican que todos los entrevistados realizan su programación en equipo, ya sea con sus compañeros de ciclo y concretada con los de nivel, o con los especialistas del área. Ninguno de los docentes realiza la programación individualmente, a pesar de que cuatro docentes sí señalan que parte de esta programación la concretan de forma personal, tal y como señala el Docente 4:

...la parte más general de la programación la elaboramos por ciclo, los aspectos más concretos por curso (los dos $3^{\circ}$ ) y, personalmente, teniendo en cuenta las características de mi grupo de alumnos, concreto aún más la programación elaborada.

Destacar que seis de los siete docentes elaboran la programación al inicio de curso, y que ese mismo número de docentes dicen seguir alguna de las propuestas que hace las editoriales. Cuatro señalan que adaptan lo propuesto por la editorial según los alumnos y el contexto. Mientras que solo uno dice que lo adapta según la legislación vigente.

Este hallazgo evidencia una preocupación de los docentes por contextualizar las directrices establecidas desde diferentes dimensiones (equipo de ciclo, de nivel, marco legislativo o editoriales). A la vez que se evidencia el trabajo en equipo desa- 
rrollado en este centro educativo; en contraposición con el trabajo individualizado propio de los procesos de enseñanza-aprendizaje.

Tabla 2. Categorías correspondientes a la programación

\begin{tabular}{|l|c|}
\hline Con quién se elabora la programación & Total \\
\hline Programación elaborada en equipo por ciclos & 5 \\
\hline Parte, concretada en equipos de nivel & 5 \\
\hline Programación elaborada en equipo de especialistas de área & 2 \\
\hline Parte de la programación elaborada individualmente & 4 \\
\hline Cuándo se elabora la programación & 6 \\
\hline Elaborada al inicio de curso & \\
\hline $\begin{array}{l}\text { Modificada a lo largo del curso con reuniones con los compañeros que } \\
\text { dan al mismo curso }\end{array}$ & 2 \\
\hline
\end{tabular}

\section{b) Segundo bloque: Metodología}

En el segundo bloque de la entrevista se analizan los datos correspondientes a la metodología utilizada por los docentes en el aula, así como el interés y el conocimiento de la diversidad metodológica existente. Para ello, se han realizado varias cuestiones con el fin de obtener una visión, lo más completa posible, de los métodos didácticos y las estrategias que utilizan los docentes.

Como podemos ver en la tabla 3 se percibe una gran heterogeneidad, pues aunque varios docentes coinciden en que trabajan basándose en los focos de interés y la motivación del alumno, adaptándose a las circunstancias y proponiendo una amplia diversidad de actividades, son muchas más las formas de trabajar que señalan.

Tabla 3. Categorías referentes a cómo trabajan con los alumnos

\begin{tabular}{|l|c|}
\hline Cómo trabajan con los alumnos & Total \\
\hline Basándome en sus focos de interés y de motivación, captando su atención & 5 \\
\hline Haciendo las clases dinámicas y abiertas & 4 \\
\hline Trabajo en grupo del alumnado & 2 \\
\hline Trabajo individual con cada alumno & 3 \\
\hline Tomando como referencia lo programado & 1 \\
\hline De forma flexible, me adapto a las circunstancias y a ellos & 5 \\
\hline Explicación a la clase (clase magistral) & 3 \\
\hline Proponiendo diversidad de actividades & 5 \\
\hline Que no se aburran, sorprendiéndolos todos los días & 2 \\
\hline Trabajando más en clase y mandando pocos deberes para casa & 1 \\
\hline Dentro y fuera del aula ordinaria, en colaboración con el tutor & 1 \\
\hline
\end{tabular}


Ninguno de los entrevistados utiliza nombres de metodologías concretas cuando se les pregunta sobre la que usan con mayor frecuencia y de qué depende. De hecho, los resultados constatan la confusión terminológica entre métodos y estrategias didácticas, así como la reducción de ambos conceptos a actuaciones puntuales del profesor en el aula. Generalmente señalan el trabajo en grupo o metodologías participativas en las que indican una interacción constante con los alumnos para realizar una clase dinámica. Sin embargo, sí que describen la forma en que trabajan con sus alumnos; ejemplo de ello es lo expresado por el Docente 1:

Utilizo, sobre todo, el trabajo en grupo y la interacción constante con ellos, de forma que aunque yo sea la que dirige la clase, dejo que ellos aporten ideas y propongan cosas...

Son varios los docentes que han comentado utilizar más de una metodología, dependiendo de distintas circunstancias. Así, seis de los docentes destacan la interacción constante y el dinamismo en clase con los alumnos, la utilización de una metodología más participativa, que podríamos relacionarlo con las principales características del aprendizaje cooperativo. Igualmente, los docentes utilizan la metodología expositiva o la lección magistral, el trabajo autónomo y, a modo testimonial, aparece el aprendizaje basado en problemas que utiliza uno de los docentes.

Este hecho refleja la adquisición de un nuevo modelo, obedeciendo a lo señalado por Guarro (2002) o De Miguel (2007), quienes afirmaban la no idoneidad de una única metodología para todas las situaciones de aprendizaje, ya que el reto se sitúa en ampliar el repertorio metodológico. A ello se suma que los datos revelan que las metodologías dependen, en muchos casos, de la materia impartida; datos corroborados, tanto en preguntas referentes a competencias, como a objetivos y metodologías.

Realizando un análisis vertical observamos que los especialistas promueven constantemente la interacción y la participación de sus alumnos, favoreciendo un aprendizaje más cooperativo, mientras que la mayoría de generalistas siguen una estructura similar en sus clases, más caracterizadas por ser expositivas. Aún así, la mayoría (cuatro de los docentes) han señalado utilizar distintas metodologías, dependiendo del contenido tratado, de la materia o de las características del grupo.

Los docentes dicen promover la participación de los alumnos mediante la realización de preguntas en clase, bien sobre los contenidos dados, sobre la vida personal y las experiencias del alumno o sobre sus temas de interés. Además, la mitad de ellos dicen realizar actividades de clase y trabajos en grupo que promueven dicha participación. Así, se puede afirmar que los métodos de enseñanza con participación del alumno, donde la responsabilidad del aprendizaje depende directamente de su actividad, implicación y compromiso, son conocidos y utilizados por estos docentes, ya sean generalistas o especialistas. Tal vez ésta sea la metodología de "avanzadilla" para la continuidad en el proceso de cambio metodológico propuesta en la concepción teórica de las competencias.

Si bien, este modo de trabajar con los alumnos es contradictorio con la forma de organizar a los alumnos en las clases (ver tabla 4). Debemos de recordar que, como planteaba de Sales (2005), la disposición espacial condiciona las estrategias de enseñanza-aprendizaje que se ponen en práctica. 
Tabla 4. Categorías correspondientes a la organización del espacio

\begin{tabular}{|l|c|}
\hline Organización del espacio & Total \\
\hline De diversas formas, cambio frecuente de organización del aula & 3 \\
\hline No suelo cambiar la organización del aula & 4 \\
\hline En forma de U & 4 \\
\hline En parejas & 4 \\
\hline En pequeños grupos & 2 \\
\hline Todas las mesas (6) concentradas en el centro de la clase & 1 \\
\hline Justificación de la organización de la clase & \\
\hline Dependiendo del tipo de actividades a hacer & 3 \\
\hline Dependiendo del tema o contenido trabajado & 1 \\
\hline Depende de las características y evolución de los alumnos & 3 \\
\hline Por limitaciones de espacio y por alto no de alumnos & 2 \\
\hline Permite que los alumnos interaccionen más entre ellos y trabajen en grupo & 2 \\
\hline
\end{tabular}

Así, los docentes organizan sus clases de modo diferente. La mayor parte (cuatro) mantienen la misma organización con los alumnos durante todo el tiempo de enseñanza, mientras que tres han señalado cambiarla frecuentemente; dependiendo, sobre todo, de las características y evolución a lo largo del curso de los alumnos o del tipo de actividades a realizar. El hecho de que más de la mitad de los docentes no cambien la organización del aula durante el curso corrobora lo ya dicho por Díaz Barriga (2006), cuando señala que el espacio del aula aparece abandonado en la mayoría de los casos a la rutina y al desarrollo de formas de trabajo establecidas. Generalmente la organización que mantienen los docentes es en forma de $U$, en pequeños grupos, en parejas o con las mesas en el centro de la clase, Todas permiten la interacción de los alumnos entre sí y con el docente.

Para completar la información a recoger acerca de la metodología, incluimos una cuestión acerca de cómo promueven la participación entre los alumnos y la interacción con el docente. Las respuestas permitieron establecer ocho categorías, como podemos observar en la tabla 5, que desprenden bastante unanimidad aunque con matices distintos. La mayoría de los docentes señalan promover la participación del alumnado en clase realizándoles preguntas, ya sea sobre contenidos o sobre aspectos de su vida y/o temas de su interés y, con menor frecuencia, realizando trabajos en grupo, ciertas actividades y dejando frases o situaciones inacabadas, para favorecer la interacción en clase, tal y como refleja la respuesta del Docente 1:

Sobre todo preguntando mucho a todos los alumnos o haciendo como que se me ha olvidado algo, para que ellos participen en clase y recuerden a lo que hago referencia. También hago que aporten y participen en clase dejando frases o situaciones a medias, de forma que ellos tengan que terminarlas, solucionarlas...

En conclusión, la mayoría de los docentes utilizan una metodología centrada en el aprendizaje cooperativo, promoviendo la participación e interacción con sus 
alumnos. Aquellos que lo realizan plantean preguntas y con actividades de grupo apoyándose en una organización del aula en forma de herradura, formando parejas o grupos reducidos.

Tabla 5. Categorías de cómo promueve la participación en clase

\begin{tabular}{|l|c|}
\hline Participación con y entre los alumnos & Total \\
\hline Haciéndoles preguntas sobre los contenidos dados & 6 \\
\hline Haciéndoles preguntas sobre su vida o temas de su interés & 4 \\
\hline Haciéndoles preguntas que generen debate & 1 \\
\hline Con la realización de actividades de clase & 3 \\
\hline Con la realización de trabajos en grupo & 3 \\
\hline Dejando frases o situaciones inacabadas & 2 \\
\hline Motivándoles en base a los conocimientos previos que tengan & 1 \\
\hline Dándoles responsabilidades a los que menos participan & 1 \\
\hline
\end{tabular}

Cabe recordar, que de modo habitual los profesores no suelen cambiar la distribución espacial del aula, fijada desde el inicio de la misma.

\section{c) Tercer bloque: Contenidos y Actividades}

Con relación a los contenidos se han planteado tres cuestiones acerca de las actividades que los docentes llevan a cabo en el aula, si siguen alguna secuenciación y se les ha pedido la ejemplificación de alguna de sus clases, que aporta una gran riqueza al resto de información, no solamente de este bloque, sino también del anterior.

En la tabla 6 reflejamos la variabilidad de actividades señaladas por los docentes, separando los tipos de actividades y la manera de implementarlas. Como se observa en esta tabla, los docentes utilizan tanto actividades individuales como grupales, todas ellas muy variadas. Las actividades señaladas con mayor frecuencia son: actividades del libro, trabajos manuales, fichas, lecturas y cálculo.

Sorprende observar que, a pesar de que varios docentes han señalado realizar actividades en grupo variadas y dinámicas, a la hora de apuntar tipos de actividades han citado actividades tradicionales que se suelen realizar de forma individual, como fichas, lectura y cálculo.

Todos los docentes coinciden en seguir una secuenciación fija: actividades de motivación, desarrollo, refuerzo y/o ampliación. Solamente dos profesores han explicitado realizar, adicionalmente, actividades de evaluación.

Las razones que han argüido sobre el tipo de secuenciación hacen referencia a despertar el interés de los alumnos, la necesidad de adaptación de éstas a los alumnos y al contexto o ampliación de los contenidos. Esta cierta carencia de flexibilidad en la planificación es justificada por los docentes que afirman que la secuenciación la dejan planteada al inicio de curso. 
Tabla 6. Categorías correspondientes al tipo de actividades

\begin{tabular}{|l|l|}
\hline Actividades & Total \\
\hline Tipos de actividades & \\
\hline Actividades del libro & 4 \\
\hline Trabajos manuales (murales, dibujos) & 3 \\
\hline Búsqueda de información en Internet & 2 \\
\hline Fichas & 3 \\
\hline Canciones & 2 \\
\hline Juegos & 2 \\
\hline Adivinanzas & 1 \\
\hline Asambleas & 1 \\
\hline Debates & 1 \\
\hline Lecturas & 3 \\
\hline Cálculo & 3 \\
\hline Resolución de problemas & 2 \\
\hline Redacciones & 2 \\
\hline Poesías & 2 \\
\hline Dictados y actividades de ortografía & 2 \\
\hline Danzas & 1 \\
\hline Listening & 1 \\
\hline Representaciones y role-playing & 1 \\
\hline Comentarios de texto & 1 \\
\hline Actividades de investigación & 1 \\
\hline
\end{tabular}

Cuando preguntamos a los docentes que nos explicaran tres ejemplos de sus clases, la mayoría nos indicó un sólo ejemplo, de hecho sólo uno dio dos. Todos los entrevistados señalan que siguen habitualmente la misma estructura o rutina a la hora de impartir sus clases, a la vez que 5 de ellos indican que dependiendo de la materia o del contexto puede variar un poco el planteamiento de la clase. Como se indica en la tabla 7, todos los docentes señalan acciones de la fase de inicio y de la fase de desarrollo, siguiendo la terminología de autores como Giné y Parcerisa (2003), Quinquer (2003) y Martínez, Miralles y Alfageme (2008), si bien, sólo tres indican actividades de la fase de cierre, señalando acciones o actividades de relajación y/o canciones que se realizan entre clase y clase.

En relación con la fase de inicio, los datos que podemos ver en la tabla anterior nos señalan que la acción más realizada (6 docentes) por los profesores es la del repaso o preguntar por contenidos explicados en otras sesiones de clase. Además, tres docentes dicen que al comienzo de las clases los alumnos se colocan en sus sitios, dejan las mochilas y rezan (recordemos que las entrevistas se han realizado en un Colegio Concertado Católico). Estos tres mismos profesores dicen que tratan de captar la atención 
de sus alumnos al inicio de la clase con distintos mecanismos. Como dato anecdótico indicar que sólo uno de los docentes dice saludar al llegar a clase.

Tabla 7. Categorías correspondientes a ejemplos de clases

\begin{tabular}{|l|c|}
\hline Ejemplos de clase & Total \\
\hline En general & \\
\hline Seguimos la misma estructura/rutina & 7 \\
\hline Depende de la asignatura y/o el contexto & 5 \\
\hline Fase de inicio & 7 \\
\hline Nos saludamos & 1 \\
\hline Los alumnos se colocan en su sitio y dejan la mochila & 3 \\
\hline Rezamos & 3 \\
\hline Capto su atención & 3 \\
\hline Hago preguntas sobre contenidos de la materia/ repaso & 6 \\
\hline Fase de desarrollo & 7 \\
\hline Relaciono lo que les enseño con la vida real y cotidiana & 2 \\
\hline Explico los contenidos a trabajar & 4 \\
\hline Sacan el libro y explico & 3 \\
\hline Realización de ejercicios y/o actividades & 7 \\
\hline Corregimos los deberes y/o ejercicios & 5 \\
\hline Pregunto si tienen dudas & 3 \\
\hline Realizamos esquema o subrayamos lo explicado & 4 \\
\hline Pongo deberes & 6 \\
\hline Fase de cierre & 3 \\
\hline Actividades de relajación y/o canciones & \\
\hline
\end{tabular}

En referencia a los datos recogidos sobre la fase de desarrollo, destacar que todos los docentes realizan ejercicios y/o actividades con los alumnos en clase y uno de ellos reconoce mandar tareas para casa, aunque sólo 5 de ellos dicen corregirlos. Todos los docentes, asimismo, explican los contenidos a trabajar, si bien 3 de ellos dicen explícitamente que siguen el libro de texto. Respecto a las explicaciones también nos hemos encontrado que 2 docentes dicen tratar de relacionar lo que les enseñan a los alumnos con la vida real y cotidiana; 3 preguntan en clase sobre las dudas de los alumnos y uno de ellos trabaja los contenidos explicados con sus alumnos realizando esquemas o subrayando lo explicado.

\section{d) Cuarto bloque: Evaluación}

En el apartado referente a la evaluación planteamos las preguntas relativas a: cómo se evalúa, cuándo se evalúa, con qué instrumentos y la importancia de la evaluación para el docente (ver tabla 8). 
Tabla 8. Categorías correspondientes a cómo es la evaluación

\begin{tabular}{|l|c|}
\hline Cómo es la evaluación & Total \\
\hline La evaluación es continua & 5 \\
\hline La evaluación intento que sea lo más individualizada posible & 2 \\
\hline La evaluación la realizo de forma conjunta con sus tutores & 1 \\
\hline ¿Qué se tiene en cuenta en la evaluación? & \\
\hline Teniendo en cuenta el trabajo diario, el día a día en clase & 3 \\
\hline Examen & 3 \\
\hline Evalúo el interés y el esfuerzo de cada alumno & 5 \\
\hline Su participación e interacción en las actividades & 6 \\
\hline $\begin{array}{l}\text { Las producciones de los trabajos. El orden y la limpieza de los trabajos } \\
\text { realizados y de la libreta }\end{array}$ & 3 \\
\hline El comportamiento & 3 \\
\hline La consecución de los objetivos curriculares o de intervención & 2 \\
\hline
\end{tabular}

La mayoría realizan una evaluación continua, destacando que lo que más tienen en cuenta es la participación e interacción de los alumnos en las actividades, el interés y el esfuerzo de cada alumno. La revalorización de la evaluación formativa-continua y una revisión de la evaluación final dilucidan formas de actuar más centradas en el alumno. En este sentido, suelen utilizar la evaluación estratégicamente de modo integrado en las actividades de aprendizaje y enseñanza. A su vez, señalan utilizar varios instrumentos de evaluación, coincidiendo la mayoría en la observación sistemática, la realización de controles finales y los trabajos diarios.

Cabe incidir en que los resultados reflejan una escasa preocupación por la evaluación de la práctica docente. A pesar de ser un tema la máxima actualidad introducida en el currículum vigente, solamente es tenido en cuenta por un docente, el Docente 6:

Evalúo sobre todo a través de la observación, de hojas de registro y de controles que solamente suponen una parte de la nota que hayan ido obteniendo con su trabajo diario. No condicionan totalmente la nota final. Y mi práctica docente con un cuestionario muy sencillo...

En lo que respecta a cuándo se evalúa, cinco docentes señalan varios momentos en los que realizan la evaluación, además seis indican que la evaluación se realiza de forma continua y diaria; de los cuáles solamente cuatro han señalado realizar una evaluación inicial y un control al final de cada trimestre.

Sobre este tema, nos encontramos con datos contradictorios, ya que mientras que los datos recogidos con esta cuestión reflejan que tres de los docentes realizan un control al final de cada unidad didáctica, en la cuestión anterior solamente uno mencionaba realizar controles parciales por cada unidad didáctica.

Respecto la importancia que tiene para los docentes la evaluación, podemos comprobar que los docentes indican varias finalidades, siendo la principal valorar el 
aprendizaje del alumno, comprobar que logran los objetivos y mejorar el proceso de enseñanza-aprendizaje. Con menor frecuencia aparecen finalidades como conocer a los alumnos y la mejora de la propia práctica docente.

De este modo, queda patente que la evaluación es utilizada fundamentalmente desde la concepción de "rendimiento de cuentas".

\section{CONCLUSIONES}

Los resultados obtenidos dejan constancia de lo que ocurre en las aulas del Centro Educativo estudiado. Así, podríamos concluir que aún no se ha asimilado el modelo centrado en el alumno o modelo centrado en competencias. Sin embargo, no sería aventurado decir que hay indicios de la asunción de un cambio en la práctica docente: promover en sus clases la interacción y la participación, utilizar diversas metodologías que fomentan un aprendizaje cooperativo, trabajar en grupo tanto docentes como alumnos, usar una evaluación continua, la diversidad de actividades que destacan proponer a los alumnos, etc. Todo ello parece indicar que se está iniciando el camino hacia el cambio señalado. Sin olvidarnos de que, a la vez, nos encontramos con profundas contradicciones en las actuaciones de los docentes, como el planteamiento de una secuencia fija que hace denotar cierta inflexibilidad; el diseño de material propio, que luego no mencionan utilizar en sus clases, o no cambiar la organización del aula. Esto parece incidir en el hecho de que aún nos encontramos en un estadio muy incipiente del proceso del cambio.

Somos conscientes de las limitaciones de la investigación realizada, al ser número de docentes reducido y al centrarse en un solo centro, además de que los datos obtenidos proceden exclusivamente de las entrevistas realizadas a los docentes. No obstante, éste se trata de un primer acercamiento a la realidad de las aulas de Primaria y al desarrollo de experiencias educativas que se dan dentro de ellas. Es necesario conocer qué es lo que opinan los docentes sobre su propia práctica y permitirnos reflexionar sobre la misma. Del mismo modo, permite a los investigadores como nosotros adentrarnos en las complejidades y dificultades reales que tienen los cambios y las innovaciones para poder permear en cualquier realidad educativa, dando paso con ello a un problema de mayor calado: ¿Cuál es el impacto real de todos los cambios e innovaciones que se plantean a nivel teórico o, sin más extremo, a nivel legislativo?

Sin duda, el seguir profundizando en el conocimiento de la realidad educativa es una premisa básica para cualquier profesional del ámbito educativo. Así, la continuidad de esta línea de trabajo podría ser ampliada, al realizar esta experiencia en otros centros educativos de la Región de Murcia, hecho que nos permitiría comparar datos y tener una visión más genérica sobre las metodologías empleadas en los centros de Primaria de nuestra Comunidad. Además, puesto que hemos constatado la inexistencia de trabajos sobre la realidad docente y, más concretamente, sobre las metodologías llevadas a cabo por los docentes en los centros, nos planteamos la necesidad de promover estudios relativos a este tema que conformarán una base sólida de conocimiento sobre dichas metodologías, que proporcionarían una ayuda útil para diseñar programas de formación e introducir los nuevos modelos educativos de forma contextualizada y, por tanto, con mayor impacto. 


\section{REFERENCIAS BIBLIOGRÁFICAS}

BERMEJO, V. (2006). Formación del profesorado y cambios metodológicos. Didáctica, Innovación y Multimedia, 3, 1-7.

BLASCO, T. y OTERO, T. (2008). Técnicas conversacionales para la recogida de datos en investigación cualitativa: La entrevista (II). Nure Investigación, 33. Disponible en: http://www.nureinvestigacion.es/FICHEROS_ADMINISTRADOR/ F_METODOLOGICA/formet_332622008133517.pdf. (Consultado 05/09/2009).

DE MIGUEL, M. (2005) (Coord.). Modalidades de Enseñanza centradas en el desarrollo de Competencias: orientaciones para promover el cambio metodológico en el Espacio Europeo de Educación Superior. Proyecto EA2005-0118.

DE MIGUEL, M. (2006). Metodologías de enseñanza y aprendizaje para el desarroIlo de competencias: orientaciones para el profesorado universitario ante el espacio Europeo de Educación Superior. Madrid: Alianza.

DÍAZ BARRIGA, A. (2006). El enfoque de competencias en la Educación ¿Una alternativa o un disfraz de cambio? Perfiles educativos, 111, 7-36.

ESCUDERO, J. M. (1990). Tendencias actuales en la investigación educativa: los desafíos de la investigación crítica. Qurrículum, 2, 3-26.

FERNÁNDEZ MARCH, A. (2005). Nuevas metodologías docentes. Valencia: ICE, Universidad Politécnica de Valencia.

FERNÁNDEZ MARCH, A. (2006). Metodologías activas para la formación de competencias. Valencia: ICE, Universidad Politécnica de Valencia.

GARROTE, D. (2008). Nuevos retos educativos: el modelo docente en el espacio europeo. Contextos educativos, 11, 246.

GINÉ, N. y PARCERISA, A. (Coords.) (2003). Planificación y análisis de la práctica educativa. La secuencia formativa: fundamentos y aplicación. Barcelona: Graó.

GUARRO, A. (2002). Curriculum y democracia. Por un cambio de la cultura escolar. Barcelona: Octaedro.

MARTÍNEZ-VALCÁRCEL, N., MIRALLES-MARTÍNEZ, P. y ALFAGEME-GONZÁLEZ, M. B. (2008). A Reflection on the Class Teaching Sequence with Particular Reference to History Classes in Spain. The Australian Journal of Teacher Education, $33(6), 61-75$.

MILES, M. B. y HUBERMAN, A. M. (1984). Qualitative data analysis: a sourcebook of new methods. Londres: Sage.

MURILLO, P. (2007). Nuevas formas de trabajar en la clase: metodologías activas y colaborativas. En F. Blanco (Dir). El desarrollo de competencias docentes en la formación del profesorado (pp. 129-153). Madrid: MEC-Colección Conocimiento Educativo.

PAREDES LABRA, J. (1999). Análisis etnográfico de los usos de recursos y materiales didácticos en educación primaria: estudios de los casos de dos centros. Tesis Doctoral. Universidad Complutense de Madrid.

PERRENOUD, Ph. (2004). Diez nuevas competencias para enseñar. Barcelona: Graó. 
PERRENOUD, Ph. (2008). Construir las competencias ¿Es darle la espalda a los saberes? Boletín de la Revista Docencia Universitaria, 2. Disponible en: http:// www.redu.um.es/Red_U/m2. (Consultado 21/09/2009).

QUINQUER, D. (2003). ¿Qué estoy estudiando? ¿Qué aprenderé en esta lección? Aula de Innovación Educativa, 127, 13-15.

BOE (2006). Real Decreto 1513/2006 por el que se establecen las Enseñanzas Mínimas de Educación Primaria.

RODRÍGUEZ IZQUIERDO, R. M. (2008). Un modelo de formación basado en las competencias: hacia un nuevo paradigma en la enseñanza universitaria. Contextos educativos, 11, 131-148.

SALES, C. (2005). Análisis de las estrategias de enseñanza con tecnologías de la información zun nuevo contexto metodológico en Secundaria? Tesis Doctoral. Universidad de Valencia.

SHULMAN, L. (1999). Portafolios del docente: una actividad teórica. En N. Lyons (Comp.), El uso del portafolio. Propuestas para un nuevo profesionalismo docente (pp. 56-62). Buenos Aires: Amorrortu.

VALLEJO, M. y ALFAGEME, M. B. (2009). An instrument for the evaluation on teaching. The International Journal of Learning, 16 (3), 137-150

WOOLF, H. y LOHMAN, C. (2001). Self-Initiated Learning Activities of Experienced Public School Teachers: Methods, sources, and relevant organizational influences. Tea-chers and teaching: theory and practice, 7 (1), 16-59. 